\title{
Realidad y Ser en la Filosofía Española
}

\author{
Por Jưlíán Marías
}

La coherencia de la filosofía española en lo que va del siglo XX es tanta, hay tanta afinidad sustancial en posiciones que por lo demás puedan ser distintas $y$ en ocasiones divergentes, que a veces se siente la esperanza de que ello responda a una simple coincidencia en la verdad. Vistas las cosas desde un mismo punto de vista, mejor dicho, desde una serie de puntos de vista ordenados en sucesión temporal, instalados en los respectivos niveles de varias generaciones, las perspectivas no son idénticas perero isí conexas; soñ varjas, $y$ por eso nos enriquecen y cada una agrega a las otras, pero se articulan $y$ son inteligibles en su conjunto. Si cada una de ellas, pbor si, ès sistemática, todas ellas componen un sistema histórico, distendido a lo largo del tiempo, Y que es lo que alguna vez he llamado el sistema de filiación intelectual. Nada es más confortador. Cada punto de vista individual, al engarzarse con los anteriores, los enriquece, integra y fertiliza, y a la par los corrobora y tal vez los corrige. Cada individuo ve con sus propics ojos, pero no sólo con ellos, sino también con los de los que le han precedido en la indagación. Y la razón es obvia: los ojos del hombre no se abren ex abrupto sobre las cosas, porque el hombre no nace espontánea y súbitamente aislado, sino que opera slempre desde un clerto nivel histórico; toda actividad intelectual viene de alguna parte $y$ va a otra; quiere decir con esto que a la mirada individual le pertenece, no solo la imagen que se forma en su retina, sino el camino, el movimiento de los ojos que éstos han recorrido para mirar precisamente allí. Por eso cada mirada incluye las precedentes, en una tradición viva que es precisamente la que hace posible la originalidad en su más hondo sentido, la originalidad originaria, genuina, auténtica, legitima, que no es 
la del "marcano" recién aterrizado de un platillo volante, sino la del hombre filial y paternalmente inserto en una tradición genealógica de pensamiento fecundo.

La historia empieza, por supuesto, con Unamuno. Aunque $-\mathrm{y}$ yo he insistido largamente en ello- Unamuno no fué estrictamente un filósofo, aunque él personalmente amaba la arbitrariedad y la inconexión, como la historia no las tolera, hay que partir de él si se habla de filosofía española en este tiempo; por eso, cuando hace años publiqué un libro sobre este tema, tuve buen cuidado de ponerlo en el umbral, porque sin él no se entiende la filosofía estricta que después de él -a veces contra él, pero con él siempre- apareció en nuestro país. Si se ponen juntos Del sentimiento trágico de la vida, escrito en 1912, y las $M e-$ ditaciones del Quijote, de 1914, iqué drama humano o intelectual surge de su contacto! Una meditación suficiente de la conexión entre esc:s dos libros egregios esclarecería de un solo golpe secretos profundos de la vida española y resortes muy escondidos de la filosofía europea de nuestra época. Probablemente fué el genial libro de Unamuno el que obligó a Ortega a iniciar ya su filosofía personal, a dar marcha atrás en su tema -el Quijote- para tomarlo previamente desde su raíz, es decir, desde una teoría de la realidad, comprometida por el soberano atractivo, la penetración y la irresponsabilidad del tremendo libro de Unamuno. Cuando éste acaba de oponer -con más agudeza y energía que nadie, hay que decirlo Qta razón a lavida, Ortega no puede esperar más para llegaroagsupdescubrimiento de la razón vital, provocado, alumbrado por la exasperante iluminación de las chispas que Unamuno arrancaba, a golpes, al pedernal de su mente celtibériccu.

La historia se repite en unos cuantos puntos decisivos, cuyo análisis nos llevaría lejos, pero sin el cual quedan oscuras grandes zonas de pensamiento; a veces el estímulo viene de fuera, porque la tradición personal que he llamado filiación se inserta en la tradición general del pensamiento te Europa o, si se quiere, de Occidente. Unas veces el estímulo tienə carácter de incitación positiva; otras, de reto, desafío o challenge; en ccasiones muestra un paralelismo inquietante y obliga a forzar la marcha; acaso algunas es la falta de eco la que actúa como factor de desaliento o, por el contrario, de solitaria y desdeñosa confianza.

No se olvide que un pensamiento filosófico nace siempre ligado a la situación histórica de la sociedad en que se vive y en la que se está radicado, de cuya sustancia se está hecho. Unamuno, claro es, no tenía una tradición filosófica española a su espalda, y tal vez por eso 
no pudo insertarse en una tradición general europea. Pero aun asi hay que hacer constar que par él existió un mínimo de tradición. El desdén que Unamuno sentía hacia Balmes era muy grande; considerable tnmbién el que sentía hacia los krausistas; y con todo, de unos y otros bebió, de unos y otros recibió impulsos decisivos, aunque fuesen en la forma de la insatisfacción y el descontento. Y ¿se imagina lo que fué para el joven Ortega encontrar ahí, como un promontorio, la figura ingente de Unamuno, en lugar de una llanura pelada? ¿Y lo que ha sido Ortega, a su vez, para todos los que después han ido naciendo a la filosofía? Y si, llegados al día de hoy, en lugar de mirar hacia el pasado volvemos los ojos al próximo porvenir, nos asaltan inquietantes reflexiones ; pero éstas sí que nos llevarían, resueltamente, demasiado lejos; quiero decir para hablar de ello hoy.

Prefiero detenerme en un punto único que es, ciertamente, decisivo: la insatisfacción que la filosofía española de nuestro tiempo ha sentido frente a la noción de ser, y que la harllevado a plantear -por lo menos a empezar a plantear - el problema filosófico de realidad como tal - del haber, y por tanto a buscar una metafísica que esté más allá de la ontología y pueda dar razón de ella. No sería difícil descubrir en Unamuno, por lo menos una sensibilidad para este tema. Cuando en 1904 -un cuarto de siglo justo antes de Was istu-metaphysik? - preguntaba: "Decidme:-ipor qué ha de haber mundo, y no que más bien no hubiera ni mundo ni nada? La existencia no tiene razón de ser, porque está sobre todas las razones" (Ensayose Vup.c78), landaba ceerca de la cuestión. Y lo mismo cuando contraponía la noción abstracta de sustancia a las "oscuras reminiscencias de sustancias concretas, de la sustancia del caldo, de lo sustancioso de un cocido, de lo insustancial de un escrito, de la sustancia de la carne", y refería todo ello a su origen en la sustancialidad de la persona humana que dura y perdura, y que es "lo único sustancial".

Pero donde el tema aparece inequívocamente y con todo rigor es en Ortega; está preludiado a lo largo de su obra, ya desde el primer libro; probablemente expuesto con minucia en sus cursos universitarios, de los que sólo ocasionalmente ha publicado fragmentos; en 1929 aparece formulado paladinamente en sus escritos. En julio de ese año, publica Ortega en la Revista de Occidente un ensayo titulado Filosofía pura, como anejo al folleto Kant, cinco años anterior, y que sólo era -dice- "una jaculatoria de centenario". En este estudio, Ortega intenta formalmente derivar el ser, retrotraerse a la realidad radical, en $y$ con la cual me encuentro, y que es la que obligará a pensarla en forma 
de ser. "Si en vez de definir sujeto y objeto por mutua negación -escribe Ortega-, aprendemos a entender por sujeto un ente que consiste en estar abierto a lo objetivo; mejor, en salir al objeto, la paradoja desaparece. Porque, viceversa, el ser, lo objetivo, etc., sólo tienen sentido si hay alguien que los busca, que consiste esencialmente en un ir hacia ellos. Ahora bien, este sujeto es la vida humana o el hombre como razón vital. La vida del hombre es en su raíz ocuparse con las cosas del mundo, no consigo mismo. El moi-meme de Descartes, que sólo se da cuenta de sí, es una abstracción que acaba siendo un error. El je nè suis qu'une chose qui pense es falso. Mi pensamiento es una función parcial de "mi vida" que no puede desintegrarse del resto. Pienso, en definitiva, por algún motivo que no es, a su vez, puro pensamiento. Cogito quia vivo, porque algo en torno me oprime y preocupa, porque al existir yo no existo sólo yo, sino que "yo soy una cosa que se preocupa de las demás, quiera o no". No hay, pues, un moi-meme sino en la medida en que hay otras cosas, y no hay otras cosas si no las hay para mi. Yo no soy ellas, ellas no son yo (anti-idealismo), pero ni yo soy sin ellas, sin mundo, ni ellas son o las hay sin mí para quien su ser $y$ el haberlas puede tener sentido (anti-realismo). Y agrega unas líneas más abajo: "Las cosas por sí no tienen medida, son desmesuradas, no son ni más ni menos, ni así, ni del otro modo, en suma, ni son ni no son. La medida de las cosas, su modo, su ni más ni menos, su así no de otra manera, es su ser y esté set implica la intervención del hombre".

La cosa está, pues, clara: el hombre se encuentra oprimido por lo que hay, por la realidad, y ésta lo obliga a preguntarse por ella e interpretarla desde el punto de vista del ser, con lo cual aparece la medida o "es" de las cosas, como resultado de la actividad del hombre con ellas. Ya en 1914 había escrito Ortega una frase reveladora: "En suma: la reabsorción de la circunstancia es el destino concreto del hombre".

Esta visión del problema tiene desarrollos mucho más amplios y explícitos, procedentes de la cátedra de Metafísica de la Universidad de Madrid. En los primeros meses de 1931, publicó Ortega cuatro largos artículos en El Sol, bajo el título ¿Qué es el conocimiento? (Trozos de un curso). En ellos se plantea la cuestión con todo su volumen.

Al interpretar la filosofía como algo que el hombre hace, Ortega tiene que preguntarse en qué consiste ese hacer humano que es preguntar; y esto lo lleva a la cuestión de las preguntas esenciales, cuyo esquema es "¿Qué es tal cosa?". Cuando pregunto ¿qué es la luz?, ob- 
serva Ortega, pregunto por el ser de la luz y no por la luz misma, que lengo delante y no me preocupa. "No busco las cosas, sino su ser". Este ser está ligado a la cosa, pero no es ella, está "detrás" de ella, oculto por ella. Es esta luz la que me hace preguntarme por su ser, y ella no es ella misma, no es su ser. Tengo que quitar lo patente para descubrir o desvelar lo latente -alétheia-. "La luz es una cosa; pero su ser, no - será a lo sumo una "cuasi-cosa", de donde viene la voz trivial "quisicosa". A esta cuasi-cosa en que consiste lo que una cosa es le llamaremos su "esencia"."

Y continúa Ortega su análisis: "Con esto resulta que se nos ha duplicado el mundo. Cada uno de nosotros vive rodeado de cosas, de objetos inmediatos, que se presentan y hacen patentes por sí mismos... Al conjunto de todas esas cosas que son entidades inmediatas, presentes por sí, llamamos circunstancia $\circ$ mundo. Pero ahora resulta que cada una de ellas tiene un ser, una esencia, lo cual implica una duplicación del mundo. Tras el mundo de las cosas está el mundo de las esencias. Tras los entes, el orden/constituído por el ser de esos entes." Y ahora viene lo más importante:

"El mundo de las cosas o entes es inmediato, está ahi entre nosotros, no tenemos que preguntarnos por él... En cambio, el mundo de las esencias, del ser, no es nunca inmediato; está siempre detrás de las cosas, mediado por éstas. Importa mucho caer en la cuenta y subrayar esta peregrina condición, tencapariencia pocosimportante, pero que a su hora resultará decisiva que el lser da esencia, es algo que no se da por sí, sino que tiene que ser buscado por el hombre, que si se encuentra, es al cabo de un esfuerzo a veces penosísimo. Precisamente lo contrario de lo que acaece con las cosas, las cuales no sólo no hay que buscarlas originariamente, sino que se anticipan a toda ocupación nuestra con ellas; mús aún: se anticipan a nuestra vida misma. Pues es importantísimo notar que vivir es ya de suyo primordial y necesariamente encontrarse cada uno entre las cosas, frente a ellas, rodeado y sumergido en ellas..." "Consecuencia de lo anterior. Si el existir del hombre es necesariamente existir entre cosas, quiere decirse que el hombre necesita absolutamente de las cosas. En cambio, el ser, las esencias, necesitan del hombre, por lo menos y por lo pronto en el sentido de que necesitan ser buscados por él".

Las consecuencias de este punto de partida no se hacen esperar. Una nueva idea de metafísica se va perfilando: "¿No indica ya esto que el ser es algo que está en la pregunta del hombre -quiero decir que consiste en ser pregunta-, en un hacer del hombre? Si no exis- 
tiese alguien capaz de preguntar qué es esto o lo otro, ¿existiría el ser?" $Y$, iras otras muchas cosas, concluye este artículo: "El mundo inmediato es el que hallamos sin buscarlo, lo que encontramos tan primordialmente, que encontrarlo no supone un acto mental especializado, sino que encontrarlo es una y misma cosa con nuestra existencia. Vivir es, en efecto, hallarse entre las cosas y frente a ellas." (El Sol, 18-I-1931).

Esto es sólo el punto de partida. Ortega hace una crítica de la idea aristotélica según la cual el hombre conoce por naturaleza, es decir, porque tiene una facultad natural para ello. La teoría de las facultades y su uso vital es en gran parte el contenido del segundo artículo; y ella lo lleva a atacar por segunda vez el problema del ser y la realidad: "Del trasmundo del ser no nos dan las cosas de este mundo inmediato la menor noticia. El mundo no tiene poros ni agujeros, como una decoración vieja, que nos permite entrever el fondo del escenario. El mundo es un área toda patente y sin intersticios. En el mundo no hay nada del ser, presente como un dato. El ser, en cuanto tal, no se manifiesta, lo que no aparece, lo que ni en todo ni en la más mínima de sus porciúnculas se hace presente, aquello de que no tenemos la menor noticia. El ser es, en suma, lo ausente por excelencia." "Originariamente, el ser no es una cosa que está ahí, más o menos a la mano, entre las cosas, como una perla en el granero de trigo; el ser está originariamente sólo en la pregunta que por él se hace el hombre." Y esta pregunta, añade Ortega, ha de colocarse en la situación vital donde se produjo" (EI Sol, 254-193lo.Puccinelli Converso"

Esto lleva a Ortega a un nuevo problema filosófico: el del hablar y el preguntar, y a la noción de verdad como atributo de las cosas o autentícidad. El parecer de las cosas me remite a lo que son en su verdad. Si el ser o esencia se manifiestan en la pregunta, hay que averiguar por qué se tiene alguna noticia de ellos, "cómo es que hablábamos del ser, no obstante carecer de todo dato inmediato y directo sobre él" (El Sol, I-II-1931).

Nuestra vida consiste en que tenemos que sostenernos en medio de las cosas, y para ello decidir lo que vamos a hacer y ser en el instante inmediato. Tenemos que acertar, y necesitamos anticipar las cosas "mediante una imagen o esquema en que se nos revele su contextura definitiva". "No nos basta con esta luz que ahora nos alumbra, que ayer nos alumbró. Necesitamos estar seguros de si mañana nos alumbrará, y para ello nos es preciso saber a qué atenernos respecto a la luz de slempre, o lo que es igual, necesitamos descubrir la esencia o 
ser de la luz." "Esto nos hace caer en la cuenta -continúa Ortegade lo que significa originariamente el ser, la esencia de una cosa; es simplemente aquella imagen de ella que nos da seguridad vital respecto a ella... El ser es seguridad para el hombre, claridad de atenimiento frente a cada cosa, frente a su enjambre o mundo".

Las precisiones, a partir de este momento, se acumulan en el texto orteguiano: "El ser no tiene sentido más que referido a un sujeto que, como el hombre, ha manester de él. Más aún: consiste exclusivamente en una necesidad radical del hombre." "En la vida del hombre, el contorno es más poderoso que el hombre, precisamente porque una do sus partes -el futuro- no está ahí. Y el futuro es infinito no ya en tiempo $\mathrm{y}$ en cantidad, sino en calidad. Es lo indefinido, misterioso, informe, inminente. Por eso el hombre necesita reducir la infinidad o ilimitacón del mundo en que se encuentra viviendo a la dimensión finita y limitada de su vida. Es decir, tiene que forjar un escorzo finito de la infinitud. Tiene que saber hoy lo que las estrellas son siempre. Ese escorzo es el ser. El ser de algo es su siempre proyectado en una mente que dura sólo un rato. Según esto, tiene el famoso ser un carácter puramente intrahumano, doméstico. Fuera del hombre no hay ser (tal vez, tal vez -andemos con cuidado- haya que contar como un casi-hombre al animal). Por eso no está ahí; antes bien, para que lo haya tiene el hombre que buscarlo. En esta busca nace pacientemente el ser."

La doctrina no puédel isertmásataxativa ${ }^{2}$ rtega sale, sin embargo, al paso de algunos posibles malentendidos. "Esta idea -añade- de que el ser de las casos es algo que el hombre construye porque lo necesita, y consecuentemente, que no ha lugar a hablar de un ser si se abstrae de la vida humana, no implica lo más mínimo recaída en el idealismo, y menos en el que fuera peor de todos: en un idealismo antropológico. Porque aquí no se dice que las cosas, que las "realidades", sean construcción de la mente. Todo lo contrario. Porque las cosas nos aprietan inexorablemente antes de que pensemos en ellas nos vemos obligados a buscarles un ser y a descubrir y construir éste. Lo construído no son, pues, las cosas, sino su ser."

"Ahora se comprende - concluye Ortega- por qué el entendimiento funciona. No simplemente porque lo tengamos. Funciona, como en el náufrago los brazos, para mantenerlo a flote; pensar es un movimiento natatorio para salvarse de la perdición en el caos. Si se quiere insistir en la comparación, dígase que el ser es la balsa que el náufrago se construye con lo que lo rodea. El ser de una cosa no es, pues, una cosa ni una hipercosa; es un esquema intelectual. Su contenido 
nos expresa o descubre lo que una cosa es. Y "lo que una es" está constituído siempre por el papel que la cosa representa en la vida, por su significación intravital." (El Sol, I-III-1931).

La minuciosidad con que he citado estos textos me permile ser muy breve al citar otros posteriores en que Ortega recoge y desenvuelve esta teoría del ser y la realidad (1). En la primavera de 1933, Ortega dió en la Universidad de Madrid su curso En torno a Galileo; la VII de estas lecciones se publicó en la revista Cruz y Raya (octubre de 1933), bajo el título La verdad como coincidencia del hombre consigo mismo. En ella, Ortega vuelve a plantear la cuestión. Critica que las grandes filosofías del pasado hayan partido, por lo general. de que las cosas, además de su papel inmediato con nosotros, tienen un ser, y de que el hombre tiene que ocuparse en descubrirlo. Ortega pide una justificación de esto, una razón para que me interese por el ser. Lo problemático es que las cosas tengan ellas por sí un ser. "Puede acaecer -escribe- que la verdad sea todo lo contrario de lo que hasta ahora se ha supuesto: que las cosas no tengan ellas por sí un ser, $\mathrm{y}$ precisamente porque no lo tienen el hombre se siente perdido en ellas, náufrago en ellas y no tiene más remedio que hacerles él un ser, que inventárselo. Si así fuese, tendríamos el más formidable vuelco de la tradición filosófica que cabe imaginar. ¿Cómo? ¿El ser —que parece significar lo que ya está ahí, lo que ya es- consistiría en algo que hay que hacer $y$ quel por tener irremediablemente que hacerlo es la vida del hombre tan fatigosa tan laboriosa tan hacendosa?" "El ser de las cosas consistiría, según ésto, en la fórmula de mi atenimiento con respecto a ellas."

Hace pocos meses, un escritor hispanoamericano escribia en una revista española afirmaciones como éstas: "El problema de la filosofía contemporánea es completamente clásico: rehacer la pregunta que interroga por el sentido del ser... Toda la filosofía contemporánea se lanzó precipitadamente por este "Camino del ser" y no hay más que abrir las grandes obras filosóficas de nuestro tiempo (Sartre, Jaspers...) para encontrarse con una detallada y extensa discusión acerca del sentido del ser... Frente a este criterio compartido unánimemente por los mejores pensadores de nuestro siglo, Zubiri sospechó hace ya muchos años que se estaba desconociendo y pasando por alto una ins-

(1) Tengo que agradecer a mi compañera en la Universidad de California, Miss Anna Krause, el haber podido releer aquí los fragmentos de curso que oí en Madrid como alumno de Ortega. 
tancia previa y aún más radical que la del sentido del ser. Esta nueva posición suya aparece en forma escrita por primera vez en su famoso estudio sobre el problema de Dios y como el pasaje es de suma gravedad lo citaremos íntegramente: "El entendimiento conoce si algo o no es; si es de una manera o de otra; por qué es como es y no de otra manera. El entendimiento se mueve siempre en el "es". Esto ha podido hacer pensar que el "es" es la forma primaria como el hombra entra en contacto con las cosas. Pero esto es excesivo. Al conocer el hombre entiende lo que hay y lo conoce como siendo. Porqua el ser supone siempre el haber". Este texto de Zubiri -continúa el autor uruguayoque se hará clásico en la historia de la filosofía contemporánea introduce una esencial modificación en el orden de la fundamentación. Ahora sabemos que el ser no es la instancia última a que cabe llegar porque el ser está ya fundado y se funda en el habər, en lo que hay; en la realidad. La comprensión no puede ser ya definitoria del hombre, puesto que antes de comprender debemos encontrarnos con cosas reales, con cosas que "hay". En efecto: ¿cómo comprenderíamos si no nos encontráramos previamente con cosas que comprender? ... Zubiri es el primer filósofo que ha logrado ir más allá del ser y de su comprensión, más allá del plano del sentido y de las significaciones y por tanto el primer filósofo que ha superado la fenomenología. Esta proeza intelectual constituye el significado histórico de la filosofía de Zubiri y el fabuloso avance que ha realizado-sobre el resto de la filosofía europea actual." $Y$ el artículo termina con un párrafo medianamente agresivo, que prefiero no comentar" ( $\mathrm{Q})$ ?

Ahora bien, ¿qué significa esto? Simplemente, que el autor de ese artículo desconoce el pensamiento de Zubiri, tanto como la filosofía española pensada y escrita antes que él y al mismo tiempo que él. El espléndido ensayo de Zubiri En torno al problema de Dios se publicó en la Revista de Occidente en noviembre de 1935; recuerdo con emoción haber oído su lectura, de labios de su autor, y por dos veces, una en borrador y otra con el texto ya definitivamente redactado. Recuerdo también mi entusiasmo y mi deslumbramiento. Hace ya catorce años que escribí de ese ensayo: "Es un breve escrito de treinta páginas, de excepcional densidad intelectual, que representa -aunque aún no conste esto de un modo suficiente - un paso decisivo en la filosofía." Pero, naturalmente, el enorme valor y la originalidad de ese escrito de $\mathrm{Zu}$ -

(1) Alberio del Campo: "Haber y ser en la filosofía de Zubiri". (Revista, Barcelona, 23-29 de septiembre de 1954). 
biri no están en el párrafo que Alberto del Campo cita. Zubiri parte de ahí -la frase anterior, que empieza el párrafo, dice "El hombre, en efecto, tiene, entre otras, una capacidad de conocer"-, de una tesis que, aunque reciente, no era nueva, de un planteamiento del problema dentro de cuya área se movía, en fecunda convivencia intelectual, para ir a otra cosa; mejor dicho, a otras varias cosas. Una de ellas, y no de las menores, la idea de que, no ya el ser, sino el haber de Dios es peculiar y no puede confundirse con el de ninguna otra realidad, porque no es que simplemente haya Dios, sino que su modo de haber es "hacer que haya". Esto hace que el mismo "haber" sea distinto para las cosas -están ahí, las hay-y para la Divinidad - hace que haya haber-. Lo cual, a su vez, pone a Dios en una relación con la existencia humana que no puede ser la de las cosas, que no se puede reducir a la noción del "encuentro". Esto sí es original y propio de Zubiri, está radicado en una fecunda tradición y puede ser fecundo. ¿Qué significa querer reducir "el significado histórico de la filosofía de Zubiri" a un párrafo del cual parte para ir a sus propias intuiciones? ¿No significa literalmente anularlo?

Veo en ello, por el contrario, lo que decía al principio: la espléndida coherencia de la filosofía española - de la filosofía, se entiende, no de sus sucedáneos- en lo que va de siglo, la coincidencia en los problemas y en la verdad.

Y esta distinción lentre tealidad e haber yeser, desde la cual se puede avanzar en tantas direcciones; que ha permitido a Ortega llegar a las precisiones de sus Apuntes sobre el pensamiento, y a Zubiri a la más penetrante comprensión de la filosofía griega de que tengo noticia -véase, para hablar de textos escritos, Grecia y la pervivencia del pasado filosófico o Sócrates y la sabiduría griega- y el paso más audaz que se ha dado en nuestro tiempo hacia el planteamiento del problema de Dios, todavía no está sino entrevista. En el capítulo VIII de mi Introducción a la Filosofía intenté, hace ya casi un decenio, repensar y llevar algunos pasos adelante los puntos de vista de mis maestros españoles. En uno de mis últimos escritos, Idea de la Metafísica, la aplicación metódica de esa perspectiva originaria mostraba cómo la vida es la organización efectiva de la realidad, aquélla que ella tiene, en tanto en cuanto me encuentro con ella, por tanto, en la medida en que puedo llamarla la realidad en cuanto tal, frente a las teorías que operan ya desde el punto de vista del conocimiento y del ser, como las ideas de "universo", "todo de la realidad", "ente", etc. Y esa perspectiva obligaba, por último, a una idea de la metafísica como teoría 
de la vida humana $y$, por tanto, de toda realidad, pero en cuanto complicada en mi vida. Si se toma en rigor la noción de ciencia de la realidad radical - mi vida-, tiene que ser también ciencia de la radicación y de las realidades radicales, si bien sólo en cuanto radicales. Pienso que por este camino, el pensamiento español, si sabe ser fiel a sí mismo, puede llegar a importantes verdades, que naturalmente no serán españolas, sino verdades a secas, en que la realidad, descubierta $\epsilon$ interpretada, trasparece en su autenticidad.

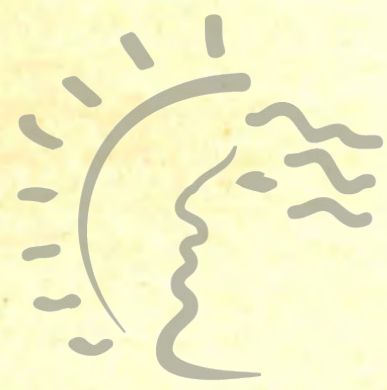

\section{Biblioteca de Letras "Jorge Puccinelli Converso"}

